\title{
Theoretical Analysis of Stiffness Constant and Effective Mass for a Round-Folded Beam in MEMS Accelerometer
}

\author{
Wai Chi Wong1,2,* - Ishak Abdul Azid ${ }^{1}$ - Burhanuddin Yeop Majlis ${ }^{3}$ \\ ${ }^{1}$ School of Mechanical Engineering, Sains University Malaysia \\ 2Department of Mechanical Engineering, College of Engineering, Tenaga Nasional University, Malaysia \\ 3 Institute of Microengineering and Nanoelectronic, Kebangsaan University Malaysia
}

In this paper, the governing equations of stiffness constant and effective mass for a round folded suspension beam in Micro-Electro Mechanical System (MEMS) accelerometer are derived and solved. The stiffness constant is determined by the strain energy and Castigliano's displacement theorem, whereas the effective mass is determined by the Rayleigh principle. The stiffness constant and the effective mass are solved separately by components and then combined by using the superposition method. The results obtained by the derived equations agree well when compared with the finite element results for several thickness values. The governing equations derived in this paper can be used to predict the natural frequencies and sensitivity of the MEMS-accelerometer.

(C)2011 Journal of Mechanical Engineering. All rights reserved.

Keywords: effective mass, folded beam, MEMS-accelerometer, stiffness constant, strain energy

\section{INTRODUCTION}

The development of MEMS devices has become increasingly important since the beginning of the 1990s. MEMS-accelerometers are the most commercially successful MEMS devices [1] and [2]. Among these, the comb finger type capacitive accelerometer is the most successful type of MEMS accelerometer due to its high sensitivity, low drift, stable dc-characteristics, low power dissipation, high bandwidth, simplicity of the fabrication process, absence of exotic materials, and low temperature sensitivity [2] to [5].

Stiffness constant and resonant frequency, which are the topics of this research, are two of the most important functionalities required in the design of any MEMS devices with moving parts. Sensors and actuators in particular, often require a specific stiffness constant and resonant frequency to guarantee successful and repeatable performance. The stiffness constant and effective mass, which incorporate both material properties and physical geometry, characterize the sensitivity and resonant frequency of the MEMS accelerometer. The sensitivity of the accelerometer is a measure of displacement with respect to acceleration. In contrast, resonant frequency characterizes the bandwidth of the accelerometer. The suspension beam can be of different designs depending on the application of the MEMS accelerometer. However, the study on the structural analysis of suspension beam in the comb finger type accelerometer has not been established well yet. Analytical derivation of these two parameters is only available for simple designs, such as straight beams [5] to [11].

For different geometric shapes, Legtenberg et al. [8] and Zhou and Dowd [9] determined the material properties of the suspension beam and the stiffness constant by using Hooke's law and the total potential energy, respectively. Tay et al. [10] used the Rayleigh's energy principle for the determination of resonant frequency as a function of effective mass. Wittwer and Howell [11] used Castigliano's displacement theorem for the analysis of the vertical deflection due to an applied moment or shear force and the geometric shape. As far as the authors know, the formula for the round folded beam has not yet been derived. Therefore, there is a need to derive the stiffness constant theoretically for a round folded beam to predict the performance of this type of design.

In this paper, a detailed derivation of the stiffness constant, as well as the effective mass for the round folded beam as used for the suspension beam in MEMS accelerometer, is carried out. The principle of the strain energy and Castigliano's displacement theorem is extensively used in 
the derivation. From the derived equations, the stiffness constant and effective mass for various parameters are determined and compared with the finite element simulation using ANSYS ${ }^{\circledR}$ 8.1.

\section{EQUIVALENT STIFFNESS CONSTANT OF THE SUSPENSION BEAM IN MEMS ACCELEROMETER}

A schematic design of MEMS accelerometer with the round folded beam is shown in Fig. 1a. The close up of the round folded beam with the symbol used in derivation is shown in Fig. 1b, where $L$ is the length of the beam, $r$ is the radius of the round part, and $w$ is the width of the beam.
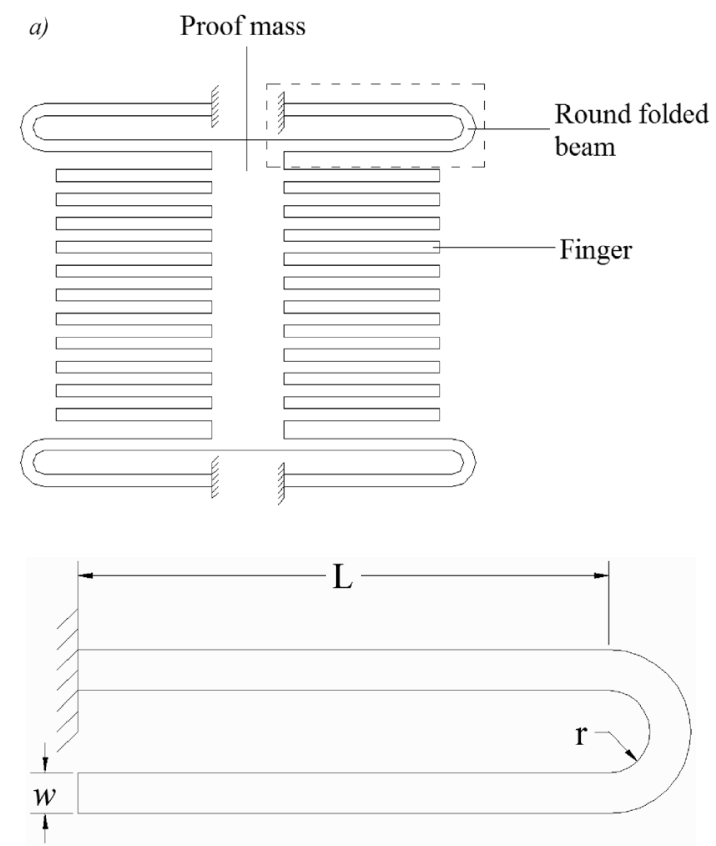

Fig. 1. Round folded beam as the suspension beam in accelerometer, a) a $2 D$ schematic diagram of MEMS accelerometer, b) the round folded beam of suspension beam

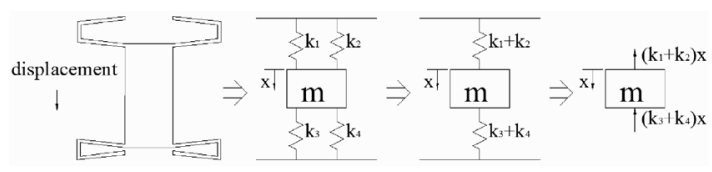

Fig. 2. Free body diagram of typical arrangement of an accelerometer
In Fig. 1a, the proof mass is suspended by four suspension beams symmetrically at the four edges. The proof mass can be approximated by a central proof mass supported by four springs. The free body diagram of the typical arrangement of an accelerometer can then be approximated by a system of mass and spring as shown in Fig. 2.

In Fig. 2, $m$ is mass of the proof mass; $k_{1}$, $k_{2}, k_{3}$, and $k_{4}$ are the stiffness constants of each suspension beam; and $x$ is the displacement. In this spring-mass system, the mass is supported by four springs, thus the external forces are balanced by the four springs evenly and stored as the strain potential energy. The equivalent stiffness constant of the spring-mass system as shown in Fig. 2 can be determined by the following equation of equilibrium:

$$
\begin{gathered}
\sum F_{x}=m \ddot{x}, \\
-\left(k_{1}+k_{2}\right) x-\left(k_{3}+k_{4}\right) x=m \ddot{x}, \\
m \ddot{x}+k_{e} x=0,
\end{gathered}
$$

where $\ddot{x}$ is the acceleration and $F_{x}$ is the force.

Therefore, the equivalent stiffness constant, $k_{e}=k_{1}+k_{2}+k_{3}+k_{4}$.

Since the four suspension beams are in the same dimension and of the same material, then

$$
k_{1}=k_{2}=k_{3}=k_{4}=k_{1 / 4}, k_{e}=4 k_{1 / 4},
$$

where $k_{1 / 4}$ is the stiffness constant of a quarter system.

In obtaining the governing Eqs. of the suspension beam for the stiffness constant, the following assumptions are made:

- The proof mass and comb fingers of the structure are rigid.

- The flexible members are attached to perfectly rigid supports.

- The device only vibrates in the sensing axis.

- Young's modulus is constant.

- Damping is ignored.

- 3D effects such as fringing field and comb finger end effects are neglected [12] to [14].

- Euler-Bernoulli beam model assumption is applied.

\section{DETERMINATION OF STIFFNESS CONSTANT IN ROUND FOLDED BEAM}

The resolved components of the round folded beam are shown in Fig. 3. The quarter 


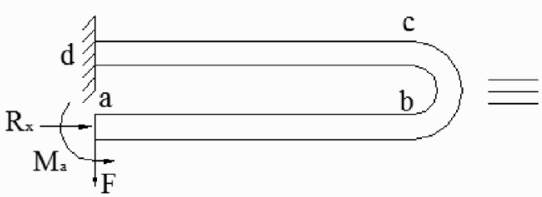

(a) The beam

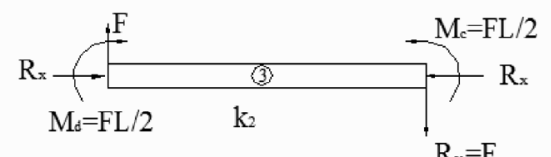

(c) Free body diagram $\mathrm{R}_{\mathrm{y}}=\mathrm{F}$ of component 3

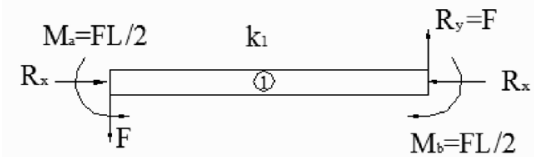

(d) Free body diagram of component 1

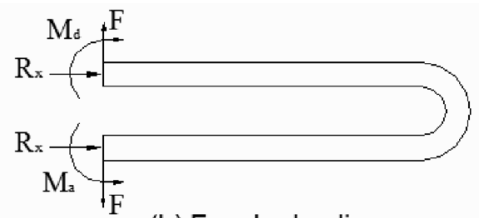

(b) Free body diagram

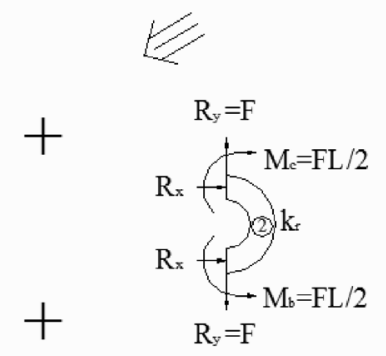

(e) Free body diagram of component 2

Fig. 3. Resolved components of theround folded beam

model of the suspension beam together with its boundary condition is shown in Fig. 3a, and its free body diagram is shown in Fig. 3b. In the analysis, the round folded beam can be resolved into three components, with two models of half fixed-fixed beam (Fig. 3c and d) and a model of half ring (Fig. 3e).

Since the device is assumed to vibrate only in the sensing axis, the total displacement of the quarter model $\delta_{1 / 4}$ is equal to the sum of the displacement of each component, $\delta_{1 / 4}=\delta_{c 1}+\delta_{c 2}$ $+\delta_{c 3}$.

Based on Hooke's law (i.e., $F=k \delta$ ), there is $k \propto \frac{1}{\delta}$. In this context, the stiffness constant of the quarter model can be given in a complementary form by:

$$
\frac{1}{k_{1 / 4}}=\frac{1}{k_{c 1}}+\frac{1}{k_{c 2}}+\frac{1}{k_{c 3}}
$$

where $k_{c 1}, k_{c 2}$, and $k_{c 3}$ are the stiffness constants for components 1 to 3 , respectively.

\subsection{The Stiffness Constant for the First and Third Components}

The free body diagram of the first and third components are similar to the model of half fixedfixed beam subjected to the transverse loading, as shown in Fig. 4. Fig. 4a shows a fixed-fixed beam with length $2 L$ subjected to a transverse load $F$ at the midspan of the beam. This force causes bending, which could result in reactions at both fixed ends consisting of forces and moments. The maximum displacement $\delta_{\max }$ occurs at the midspan of the beam. If this model is cut through its midspan, this part can be modeled as a half fixed-fixed beam. The reactions at both fixed ends are the bending moment $M_{0}$, shear reaction force in y-direction $R_{y}$, and the axial reaction force in $\mathrm{x}$-direction $R_{a}$, as shown in Fig. 4b. Since the load is transverse to the axis of the beam, the axial reaction force $R_{a}$ is extremely small compared to the bending moment and shear force. Therefore, $R_{a}$ is ignored in the calculation. The shear reaction force $R_{y}$, and bending moment $M_{0}$ for the model of the half fixed-fixed beam is obtained as $R_{y}=F / 2$ and $M_{0}=F L / 4$.

The maximum displacement in the model of the half fixed-fixed beam $\delta_{\max }$ is caused by both displacement due to bending moment $\delta_{b m}$, and the displacement due to shear $\delta_{s}$, or $\delta_{\max }=\delta_{b m}+\delta_{s}$. Thus, the stiffness constant of the half fixed-fixed beam is taken as:

$$
\frac{1}{k_{h a l p}}=\frac{1}{k_{b m}}+\frac{1}{k_{s}} .
$$

Therefore, the stiffness constant for components 1 and 3 is equal to the stiffness 
constant of the half fixed-fixed beam as given in Eq. (5a) as:

$$
\frac{1}{k_{c 1}}=\frac{1}{k_{c 3}}=\frac{1}{k_{\text {halp }}}=\frac{1}{k_{b m}}+\frac{1}{k_{s}} .
$$

\subsubsection{The Stiffness Constant Due to Bending Moment}
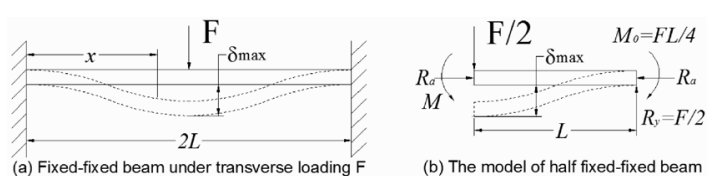

Fig. 4. Fixed-fixed beam

For a fixed-fixed beam (Fig. 4), the maximum deflection due to a bending moment occurs at the middle of the beam. It is given by [15]:

$$
\delta_{b m}=\frac{F(2 L)^{3}}{192 E I}=\frac{F L^{3}}{24 E I} .
$$

The stiffness constant due to the bending moment $k_{\text {full }}$ for the full model of the fixed-fixed beam is then derived as:

$$
k_{f u l l}=\frac{F}{\delta_{b m}}=\frac{24 E I}{L^{3}} .
$$

Thus, the stiffness constant due to the bending moment $k_{b m}$ for the half model of a fixedfixed beam is:

$$
\frac{1}{k_{b m}}=\frac{L^{3}}{12 E I}
$$

where $E$ is the Young's modulus, $I$ is the second moment of the cross sectional area, and $L$ is the length of the half model of the fixed-fixed beam.

\subsubsection{The Stiffness Constant Due to Transverse Force}

For a rectangular cross sectional area of width $b$ and depth $d$, with the total beam length $L$, and with the applied transverse load of $F / 2$, as shown in Fig. 4b, the maximum deflection (at middle point) due to the shear is given as [15]:

$$
\delta_{s}=\frac{3}{5} \frac{F_{s} L}{b d G},
$$

where $G$ is shear modulus, $G=\frac{E}{2(1+\mu)}$ and $\mu$ is the Poisson's ratio. By knowing $F_{s}=F / 2$ and by replacing $G$ and $F_{s}$ in $\delta_{s}$, the maximum deflection (at middle point) due to the shear is:

$$
\delta_{s}=\frac{6}{5} \frac{(1+\mu) F L}{b d E} .
$$

Since $k_{s}=F / \delta_{s}$, stiffness constant due to shear $k_{s}$ in the complementary form is given as

$$
\frac{1}{k_{s}}=\frac{\delta_{s}}{F}=\frac{6}{5} \frac{(1+\mu) L}{b d E} .
$$

\subsection{The Stiffness Constant for the Second Component}

The second component is approximated to be the model of a half ring with simple support on the left, transferred from the first and third components. The free body diagram is shown in Fig. 5a. This component is subjected to two forces, the transverse force $R_{y}$ and bending moment $M$. The stiffness constant of the second component $k_{c 2}$ is the combination of the stiffness constant due to transverse force $k_{t}$ (Fig. 5b), and stiffness constant due to bending moment $k_{m}$ (Fig. 5c). $k_{t}$ is approximated to be half of a stiffness constant in the ring $k_{c}$ due to the same value of transverse force, as shown in Fig. 6.

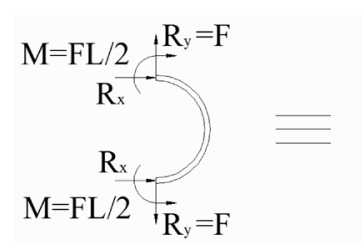

a)

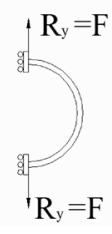

b)

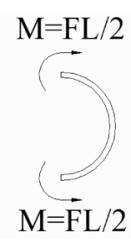

c)
Fig. 5. Free body diagram of the second component of round folded beam

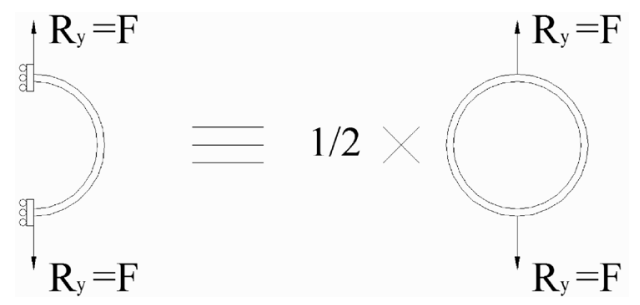

Fig. 6. Transverse force in the second component 


\subsubsection{The Stiffness Constant Due to Transverse Force Acting on Half a Ring}

The stiffness constant of the ring component (Fig. 5b) due to transverse force can be determined by using Castigliano's displacement theorem. The theorem states that deflection at a point on a member in the direction of a force applied at the specific point is given by the partial derivative of the complementary energy with respect to the external force at the point, or $\delta_{c}=\partial U / \partial R$, where $U$ is the internal strain energy given by $U=\int_{V} \frac{1}{2} \frac{\sigma^{2}}{E} d V$. For a beam subjected to bending, the direct stress is $\sigma=M y / I$, where $M$ is the bending moment, $y$ is the distance from the neutral axis to the point in question, and $I$ is the second moment of the cross sectional area $I=\int_{A} y^{2} d A$.

Thus, $U=\int_{0}^{L} \int_{A} \frac{M^{2}}{2 E I^{2}} y^{2} d A d x=\int_{0}^{L} \frac{M^{2}}{2 E I} d x$, where $L$ is the length of the beam. Accordingly, $\delta_{c}=\frac{\partial U}{\partial R}=\frac{\partial}{\partial R} \int_{0}^{L} \frac{M^{2}}{2 E I} d x$.

For a full ring, the total length is given as $L=\int_{0}^{2 \pi} r d \phi=4 \int_{0}^{\pi / 2} r d \phi$, where $r$ is the radius of the ring, and $\phi$ is the angle at any point within the ring. Since the integration of $\int_{0}^{2 \pi} d \phi$ is always zero, and in view of the symmetry of the ring, only one quadrant of the ring needs to be considered as shown in Fig. 7.

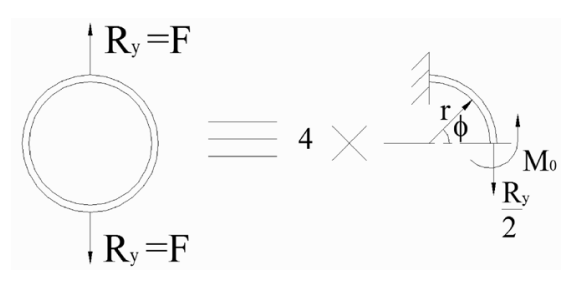

Fig. 7. Transverse forces at a ring

According to Castigliano's displacement theorem, deflection of the ring at any point $c$ is:

$$
\begin{aligned}
\delta_{c} & =\frac{\partial U_{c}}{\partial R_{y}}=\frac{\partial}{\partial R_{y}}\left(4 \int_{0}^{\pi / 2} \frac{M_{c}^{2}}{2 E I} r d \phi\right)= \\
& =4 \int_{0}^{\pi / 2} \frac{M_{c}}{E I} \frac{\partial M_{c}}{\partial R_{y}} r d \phi .
\end{aligned}
$$

By cutting the ring at any section within the quadrant of the ring, the bending moment at any point $c$ with an angle of $\phi$ is given as [15]:

$$
M_{c}=M_{0}-\frac{R_{y} r}{2}(1-\cos \phi),
$$

where $M_{0}$, the imaginary bending moment, is unknown but may be obtained from the strain energy.

The strain energy for the quadrant of the ring is:

$$
\begin{aligned}
U_{q} & =\int_{0}^{\pi / 2} \frac{1}{2 E I} M_{c}^{2} r d \phi= \\
& =\int_{0}^{\pi / 2} \frac{1}{2 E I}\left[M_{0}-\frac{R_{y} r}{2}(1-\cos \phi)\right]^{2} r d \phi .
\end{aligned}
$$
by:

$$
U_{c}=4 U_{q}=4 \int_{0}^{\pi / 2} \frac{1}{2 E I}\left[M_{0}-\frac{R_{y} r}{2}(1-\cos \phi)\right]^{2} r d \phi,
$$

owing to the symmetry at $\phi=0, \partial U / \partial M_{0}=0$. Therefore,

$$
\frac{1}{E I} \int_{0}^{\pi / 2}\left[M_{0}-\frac{R_{y} r}{2}(1-\cos \phi)\right] r d \phi=0 .
$$

Since $1 / E I \neq 0$ and $\mathrm{r} \neq 0$,

$\int_{0}^{\pi / 2}\left[M_{0}-\frac{R_{y} r}{2}+\frac{R_{y} r}{2} \cos \phi\right] d \phi=0 \quad$ solved as $M_{0}\left(\frac{\pi}{2}\right)=\frac{R_{y} r}{2}\left[\frac{\pi}{2}-(1-0)\right]$. Therefore,

$$
M_{0}=R_{y} r\left(\frac{1}{2}-\frac{1}{\pi}\right)
$$

Substituting Eq. (17) into Eq. (13), the bending moment at any point $c$ within the full ring, is:

$$
M_{c}=R_{y} r\left(\frac{1}{2} \cos \phi-\frac{1}{\pi}\right) .
$$


By partial derivative with respect to $R_{y}$ :

$$
\frac{\partial M_{c}}{\partial R_{y}}=r\left(\frac{1}{2} \cos \phi-\frac{1}{\pi}\right)
$$

Substituting Eqs. (18) and (19) into Eq. (12),

$$
\begin{aligned}
\delta_{c} & =4 \int_{0}^{\pi / 2} \frac{M_{c}}{E I} \frac{\partial M_{c}}{\partial R_{y}} r d \phi \\
& =\frac{4}{E I} \int_{0}^{\pi / 2} R_{y} r\left(\frac{1}{2} \cos \phi-\frac{1}{\pi}\right) \cdot r\left(\frac{1}{2} \cos \phi-\frac{1}{\pi}\right) r d \phi \\
& =\frac{4 R_{y} r^{3}}{E I} \int_{0}^{\pi / 2}\left(\frac{1}{2} \cos \phi-\frac{1}{\pi}\right)^{2} d \phi \\
& \delta_{c}=\frac{4 R_{y} r^{3}}{E I}\left(\frac{\pi}{16}-\frac{1}{\pi}+\frac{1}{2 \pi}\right)
\end{aligned}
$$

Since $R_{y}=F$,

$$
\delta_{c}=\frac{4 F r^{3}}{E I}\left(\frac{\pi}{16}-\frac{1}{\pi}+\frac{1}{2 \pi}\right) .
$$

The stiffness constant of the ring is then given by:

$$
\frac{1}{k_{c}}=\frac{\delta}{F}=\frac{4 r^{3}}{E I}\left(\frac{\pi}{16}-\frac{1}{\pi}+\frac{1}{2 \pi}\right) .
$$

Since the stiffness constant for the second component is equal to the half of the stiffness constant of the full ring, $k_{t}=1 / 2 k_{c}$. Therefore, the stiffness constant for the second component due to transverse force is given by:

$$
\frac{1}{k_{t}}=\frac{2}{k_{c}}=\frac{8 r^{3}}{E I}\left(\frac{\pi}{16}-\frac{1}{\pi}+\frac{1}{2 \pi}\right) .
$$

\subsubsection{The Stiffness Constant Due to Bending Moment}

The stiffness constant due to the bending moment for this component (Fig. 5c) can be determined by using the energy method. The internal strain energy is:

$$
\begin{aligned}
U & =\int_{V} \frac{1}{2} \frac{\sigma^{2}}{E} d V \\
& =\frac{2}{2 E} \int_{0}^{\pi / 2} \int_{A} \frac{M^{2}}{I_{c 2}^{2}} y^{2} d A \cdot r d \phi=\frac{1}{E} \int_{0}^{\pi / 2} \frac{M^{2}}{I_{c 2}} r d \phi
\end{aligned}
$$

$$
\begin{aligned}
& =\frac{M^{2} r}{E I_{c 2}} \cdot \frac{\pi}{2}, \\
U & =\frac{\pi M^{2} r}{2 E I_{c 2}} .
\end{aligned}
$$

Since the total internal strain energy is equal to the external work performed, $U=1 / 2 F \delta$, then, $\frac{\pi M^{2} r}{2 E I_{c 2}}=\frac{1}{2} F \delta$.Substituting $M=F L / 2$ into the equation, $\left(\frac{F L}{2}\right)^{2} \frac{\pi r}{2 E I_{c 2}}=\frac{1}{2} F \delta$.

By rearranging the above equation, the deflection due to the bending moment is:

$$
\delta=\frac{\pi F L^{2} r}{4 E I_{c 2}} .
$$

Therefore, the stiffness constant due to the bending moment is given by:

$$
\frac{1}{k_{b m 2}}=\frac{\delta}{F}=\frac{\pi L^{2} r}{4 E I_{c 2}} \text {. }
$$

Since the width and depth of the second component is equal to the width and depth of the first component,

$$
I_{c 2}=I_{c 1}=I \text {, thus } \frac{1}{k_{b m 2}}=\frac{\pi L^{2} r}{4 E I} .
$$

\subsection{The Effective Stiffness Constant of the Round Folded Beam}

The effective stiffness constant of the round folded beam can then be determined from Eq. (3):

$$
\begin{aligned}
\frac{1}{k_{e}} & =\frac{1}{4 k}=\frac{1}{4}\left(\frac{1}{k}\right), \\
\frac{1}{k_{e}} & =\frac{1}{4}\left(\frac{1}{k_{c 1}}+\frac{1}{k_{c 2}}+\frac{1}{k_{c 3}}\right), \\
\frac{1}{k_{e}} & =\frac{1}{4 k_{c 1}}+\frac{1}{4 k_{c 2}}+\frac{1}{4 k_{c 3}}= \\
& =\frac{1}{4}\left(\frac{1}{k_{b m}}+\frac{1}{k_{S}}\right)+\frac{1}{4}\left(\frac{1}{k_{t}}+\frac{1}{k_{b m 2}}\right)+\frac{1}{4}\left(\frac{1}{k_{b m}}+\frac{1}{k_{S}}\right), \\
\frac{1}{k_{e}} & =\frac{1}{2 k_{b m}}+\frac{1}{2 k_{S}}+\frac{1}{4 k_{t}}+\frac{1}{4 k_{b m 2}} .
\end{aligned}
$$

Substituting Eqs. (8), (11), (24), and (27) into Eq. (28): 


$$
\begin{aligned}
\frac{1}{k_{e}} & =\frac{1}{2}\left(\frac{L^{3}}{12 E I}\right)+\frac{1}{2}\left[\frac{6}{5} \frac{(1+\mu) L}{b d E}\right]+ \\
& +\frac{1}{4}\left[\frac{8 r^{3}}{E I}\left(\frac{\pi}{16}-\frac{1}{\pi}+\frac{1}{2 \pi}\right)\right]+\frac{1}{4}\left(\frac{\pi L^{2} r}{4 E I}\right) \\
& =\frac{L^{3}}{24 E I}+\frac{3}{5} \frac{(1+\mu) L}{b d E}+\frac{2 r^{3}}{E I}\left(\frac{\pi}{16}-\frac{1}{\pi}+\frac{1}{2 \pi}\right)+\frac{\pi L^{2} r}{16 E I}, \\
\frac{1}{k_{e}} & =\frac{1}{E t}\left[\frac{L^{3}}{2 w^{3}}+\frac{3(1+\mu) L}{5 w}+\frac{24 r^{3}}{w_{r}^{3}}\left(\frac{\pi}{16}-\frac{1}{\pi}+\frac{1}{2 \pi}\right)+\frac{3 \pi L^{2} r}{4 w_{r}^{3}}\right] .
\end{aligned}
$$

\section{DETERMINATION OF EFFECTIVE MASS IN ROUND FOLDED BEAM}

The effective mass of the folded beam is determined by using the Rayleigh principle. By taking a beam model with a cross sectional area $A$, length $L$, the displacement at any point $x$ to be $\delta(x)$, and velocity at any point $x$ to be $d \delta(x) / d t$, the maximum displacement $\delta_{\max }$ is related to the distribution function $N(x)$ as:

$$
\delta(x)=N(x) \delta_{\max } \text { and } \frac{d \delta(x)}{d t}=N(x) \frac{d \delta_{\max }}{d t} .
$$

The effective mass is given by:

$$
m_{e}=\rho \int_{0}^{L} N^{2}(x) A(x) d x .
$$

\subsection{Effective Mass for Half Model of Fixed- fixed Beam}

Since the distribution function is independent of the applied force, the distribution function can be determined by assuming the half model of the fixed-fixed beam is deflected under a concentrated force $F$. Displacement at any point within the beam is given by James et al. [16] as:

$$
\delta(x)=\frac{F}{12 E I}\left[3 L x^{2}-2 x^{3}\right] \text {. }
$$

The maximum displacement occurs at $x=L$ thus,

$$
\delta_{\max }=\frac{F L^{3}}{12 E I} .
$$

Consequently, the distribution function is:

$$
N(x)=\frac{\delta(x)}{\delta_{\max }}=\frac{3 L x^{2}-2 x^{3}}{L^{3}} .
$$

Effective mass of half model of fixed-fixed beam under bending moment is then given as:

$$
\begin{aligned}
m_{b, e} & =\rho \int_{0}^{L} N^{2}(x) A(x) d x= \\
& =\rho A \int_{0}^{L}\left(\frac{3 L x^{2}-2 x^{3}}{L^{3}}\right)^{2} d x= \\
& =\frac{\rho A}{L^{6}}\left[\frac{9 L^{2} x^{5}}{5}-\frac{12 L x^{6}}{6}+\frac{4 x^{7}}{7}\right]_{0}^{L}= \\
& =\frac{\rho A}{L^{6}}\left[\frac{9}{5}-2+\frac{4}{7}\right] \times L^{7}, \\
m_{b, e} & =\frac{13}{35} \rho A L .
\end{aligned}
$$

Thus, the effective mass of the first component $m_{1}$ is equal to that of the third component $m_{3}$ or:

$$
m_{1}=m_{3}=m_{b, e}=\frac{13}{35} \rho A L .
$$

\subsection{Effective Mass for Half Model of Ring}

For the half model of ring under transverse force (Fig. 5), the displacement at any point within the beam is given by Eq. (20) as:

$$
\delta(x)=\frac{4 R_{y} r^{3}}{E I} \int_{0}^{\phi}\left(\frac{1}{2} \cos \phi-\frac{1}{\pi}\right)^{2} d \phi,
$$

or by expanding:

$$
\delta(x)=\frac{4 R_{y} r^{3}}{E I}\left[\frac{1}{4}\left(\frac{\phi}{2}+\frac{\sin 2 \phi}{4}\right)-\frac{1}{\pi} \sin \phi+\frac{\phi}{\pi^{2}}\right]
$$

The maximum displacement is given by Eq. (21) as $\delta_{\max }=\frac{4 R_{y} r^{3}}{E I}\left(\frac{\pi}{16}-\frac{1}{\pi}+\frac{1}{2 \pi}\right)$.

The distribution function:

$$
N(x)=\frac{\delta(x)}{\delta_{\max }}=\frac{\left[\frac{1}{4}\left(\frac{\phi}{2}+\frac{\sin 2 \phi}{4}\right)-\frac{1}{\pi} \sin \phi+\frac{\phi}{\pi^{2}}\right]}{\left(\frac{\pi}{16}-\frac{1}{\pi}+\frac{1}{2 \pi}\right)}
$$

is solved by:

$$
N(x)=\frac{\frac{1}{8} \phi+\frac{1}{16} \sin (2 \phi)-\frac{1}{\pi} \sin (\phi)+\frac{\phi}{\pi^{2}}}{\frac{1}{16} \pi-\frac{1}{2 \pi}} .
$$


Effective mass of the half model of the ring under transverse force is then given as:

$$
\begin{aligned}
m_{a, e} & =\rho \int_{0}^{L} N^{2}(x) A(x) d x= \\
& =2 \rho A \int_{0}^{\pi / 2}\left(\frac{\frac{1}{8} \phi+\frac{1}{16} \sin (2 \phi)-\frac{1}{\pi} \sin (\phi)+\frac{\phi}{\pi^{2}}}{\frac{1}{16} \pi-\frac{1}{2 \pi}}\right) r d \phi .
\end{aligned}
$$

As a result, the effective mass of the second component $m_{2}$ is:

$$
\frac{\rho A r}{6}\left[\frac{2 \pi^{7}+47 \pi^{5}-32 \pi^{3}-6144 \pi}{\pi^{2}\left(\pi^{4}-16 \pi^{2}+64\right)}\right] .
$$

Thus, the effective mass of the device is

$$
\begin{aligned}
m_{e} & =m_{1}+m_{2}+m_{3}+m_{p m}+n m_{f}= \\
& =8 m_{b, e}+4 m_{a, e}+m_{p m}+n m_{f},
\end{aligned}
$$

where $m_{p m}$ is the mass of proof mass, $m_{f}$ is the mass of finger, and $n$ is the number of fingers.

\section{COMPARISON RESULTS WITH THE FINITE ELEMENT SIMULATION}

The round folded beam in MEMS accelerometer is further analyzed by using the finite element (FE) package, ANSYS ${ }^{\circledR}$ 8.1. The three-dimensional 20-node structural solid element, SOLID 186, has been used in this analysis. The material properties and the physical geometries used in the FE simulation are shown in Table 1. The FE model meshed by the free meshing is shown in Fig. 8. The simulation result will be used for a comparison with the analytical results obtained by Eqs. (29) and (40). The resonant frequency is obtained directly from the modal analysis. The stiffness constant is determined by using a static analysis.

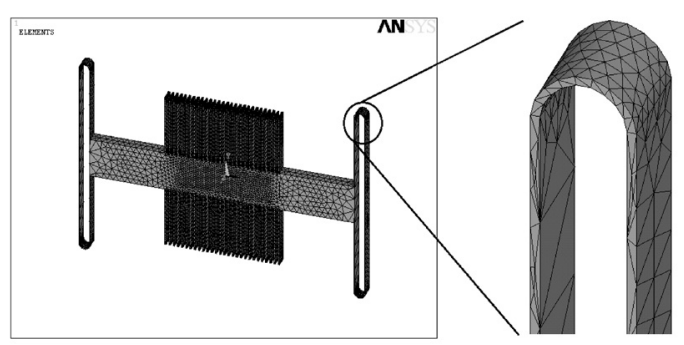

Fig. 8. Meshed model
Table 1. Material properties and physical geometries for accelerometer

\begin{tabular}{|l|c|}
\hline Elastic modulus & $\begin{array}{c}127 \times 10^{3} \\
\mathrm{~kg} / \mu \mathrm{m} \mathrm{s}^{2}\end{array}$ \\
\hline Poison's ratio & 0.27 \\
\hline Density & $\begin{array}{c}2330 \times 10^{-18} \\
\mathrm{~kg} / \mu \mathrm{m}^{3}\end{array}$ \\
\hline Length of suspension beam $(L)$ & $803 \mu \mathrm{m}$ \\
\hline Width of suspension beam $(w)$ & $11 \mu \mathrm{m}$ \\
\hline Radius of ring, $r$ & $50 \mu \mathrm{m}$ \\
\hline Length of comb finger $\left(l_{\text {finger }}\right)$ & $650 \mu \mathrm{m}$ \\
\hline Width of comb finger $\left(w_{\text {finger }}\right)$ & $15 \mu \mathrm{m}$ \\
\hline Number of comb finger & 56 pairs \\
\hline Sensing gap distance $\left(d_{0}\right)$ & $2 \mu \mathrm{m}$ \\
\hline
\end{tabular}

Table 2 shows the result of the comparison between the analytical and simulation analysis for stiffness constant and effective mass for several thicknesses $(t)$. From Table 2, it can be seen that the stiffness constant and the effective mass obtained from the derived equations based on the analytical analysis show good agreement with those obtained from the finite element simulation. The difference between analytical and simulation results is below $5 \%$. Based on this, it can be concluded that the derived analytical formulas are capable of obtaining the effective mass and the stiffness constant of the round folded beam of the suspended comb finger type accelerometer. Therefore, they are capable of predicting the resonant frequency and the sensitivity of the accelerometer.

\section{CONCLUSIONS}

The stiffness constant and the effective mass of round folded beam in MEMS accelerometer have been derived successfully. The derivation of the stiffness constant is obtained by using the strain energy method and the Castigliano's displacement theorem, while the derivation of the effective mass is determined by using the Rayleigh principle. The result obtained from these derived formulas agree well with the results obtained from FE software ANSYS.

\section{ACKNOWLEDGEMENT}

This work was carried out under the support of the short-term and the E-science grants 
Table 2. Comparison of stiffness constant and effective mass between analytical result and simulation (ANSYS) result

\begin{tabular}{|c|c|c|c|c|c|c|}
\hline \multirow{2}{*}{$t[\mathrm{~mm}]$} & \multicolumn{3}{|c|}{$k[\mathrm{mN} / \mathrm{mm}]$} & \multicolumn{3}{c|}{$m_{e f f}\left[\times 10^{-7} \mathrm{~kg}\right]$} \\
\cline { 2 - 7 } & Analytical & ANSYS & \% diff & Analytical & ANSYS & $\%$ diff \\
\hline 120 & 59.79 & 62.01 & 3.59 & 4.88 & 4.72 & -3.50 \\
\hline 80 & 39.86 & 40.46 & 1.50 & 3.26 & 3.13 & -4.01 \\
\hline 60 & 29.89 & 30.39 & 1.64 & 2.44 & 2.35 & -3.93 \\
\hline 40 & 19.93 & 19.85 & -0.42 & 1.63 & 1.58 & -3.26 \\
\hline
\end{tabular}

provided by the Universiti Sains Malaysia and the Malaysian Government.

\section{REFERENCES}

[1] Bernstein, J. (2003). An overview of MEMS inertial sensing technology. Sensors Magazine Online, vol. 20, no. 2, p. 14-21.

[2] Yazdi, N., Ayazi, F., Najafi, K. (1998). Micromachined inertial sensors. Invited Paper, Special Issue of IEEE Proc., vol. 86, no. 8, p. 1640-1659.

[3] Amini, B.V., Ayazi, F.A. (2004). 2.5V 14bit $\Sigma \Delta$ CMOS SOI capacitive accelerometer. Journal of Solid-State Circuits, vol. 39, no. 12, p. 2467-2476.

[4] Rödjegård, H., Lööf, A. (2005). A differential charge-transfer readout circuit for multiple output capacitive sensors. Sensors and Actuators A, vol. 119, no. 2, p. 309-315.

[5] Borovic, B., Liu, A.Q., Popa, D., Cai, H., Lewis, F.L. (2005). Open-loop versus closedloop control of MEMS devices: choices and issues. Journal of Micromechanics and Microengineering, vol. 15, p. 1917-1924.

[6] Chae, J.S., Kulah, H., Najafi, K. (2002). A hybrid silicon on glass lateral microaccelerometer with CMOS readout circuitry technical digest. IEEE International Conf. MEMS, Las Vegas, p. 623-626.

[7] Lüdtke, O., Biefeld, V., Buhrdorf, A., Binder, J. (2000). Laterally driven accelerometer fabricated in single crystalline silicon. Sensors and Actuators A, vol. 82, p. 149-154.

[8] Legtenberg, R. Groeneveld, A.W., Elwenspoek, M. (1996). Comb-drive actuators for large displacements. Journal of
Micromechanics and Microengineering, vol. 6, p. 320-329.

[9] Zhou, G.Y. Dowd, P. (2003). Tilted foldedbeam suspension for extending the stable travel range of comb-drive actuators. Journal of Micromechanics and Microengineering, vol. 13, p. 178-183.

[10] Tay, F.E.H., Kumaran, R., Chua, B.L., Logeeswaran, V.J. (2000). Electrostatic spring effect on the dynamic performance of microresonators. Technical Proc. International Conf. Modeling and Simulation of Microsystems, San Diego, p. 454-457.

[11] Wittwer, J.W., Howell, L.L. (2004). Mitigating the effect of local flexibility at the built-in ends of cantilever beams. Journal of Applied Mechanics, vol. 71, p. 748-751.

[12] Tang, W.C, Lim, M.G., Howe, R.T. (1992). Electrostatic comb drive levitation control method. Journal of Microelectromechanical Systems, vol. 1, p. 170-178.

[13]Li, L. Uttamchandani, D.Ë. (2006). Twinbladed microelectro mechanical systems variable optical attenuator. Optical Review, vol. 13 , no. 2 , p. 93-100.

[14] Spengen, W.M.V., Oosterkamp, T.H. (2007). A sensitive electronic capacitance measurement system to measure comb drive motion of surface micromachined MEMS devices. Journal of Micromechanics and Microengineering, vol. 17, p. 828-834.

[15] Benham, P.P., Drawford, R.J., Armstrong, C.G. (1996). Mechanics of Engineering Materials. $2^{\text {nd }}$ ed. Prentice Hall Ltd., London.

[16] James, M.L., Smith, G.M., Wolford, J.C., Whaley, P.W. (1989). Vibration of Mechanical and Structural Systems. Happer \& Row Publishers, Singapore. 